\title{
Complex Dynamics of Ishikawa Iterates for Non Integer Values
}

\author{
Yashwant S. Chauhan \\ Asst. Professor \\ Computer Science \& Engg. \\ Department
}

G. B. Pant Engg. College,

Pauri Garhwal (India)

\author{
Rajeshri Rana \\ Asst. Professor \\ Applied Science and Humanities \\ Department \\ G. B. Pant Engg. College, \\ Pauri Garhwal (India)
}

\author{
Ashish Negi \\ Asst. Professor \\ Computer Science \& Engg. \\ Department \\ G. B. Pant Engg. College, \\ Pauri Garhwal (India)
}

\begin{abstract}
We explore in this paper the dynamics of the complex function for non integer values, using the Ishikawa iterates. The $z$ plane fractal images are studied for positive powers of the complex function while c plane fractals are analyzed for negative powers of the complex function.
\end{abstract}

Keywords: Complex dynamics, Relative Superior Mandelbrot Set, Relative Superior Julia set, Ishikawa Iteration.

\section{INTRODUCTION}

In 1918, French mathematician Gaston Julia[9] investigated the iteration process of a complex function intensively and attained a Julia set, which is a landmark in the field of fractal theory. The object Mandelbrot set on the other hand was given by Benoit B. Mandelbrot [11] in 1979. The visual complexity, beauty and self similarity of these structures have made these subjects a wide area of intense research right from its advent. The various extensions and variants of both of these sets have been extensively studied using Picard's iterations. We have applied in this research article a new iteration process i.e. Ishikawa iteration.

The fractals generated from the self-squared function, $z \rightarrow z^{2}+c$ where $z$ and $c$ are complex quantities, have been studied extensively in the literature[4, 5, 6, 7 \& 10]. Recently, the generalized transformation function $z \rightarrow z^{-n}+c$ for positive integer values of $n$ has been considered by K. W. Shirriff [10]. The $z$ plane fractal images for the function $z_{n+1} \leftarrow z_{n}^{\alpha}+c$ for positive and negative, both integer and non-integer values of $n$ have been presented by Gujar et al. along with some conjectures about their visual characteristics[5, 6]. In this paper, we have considered the transformation of the functions $z \rightarrow z^{n}+c, n \geq 2$ and $z \rightarrow\left(z^{n}+c\right)^{-1}$, $n \geq 2$, and analyzed the $z$ plane fractal images for the first function and c plane fractals for the second function, generated from the iterations of these functions using Ishikawa iteration procedure. We explored the drastic changes that occur in the visual characteristics of the images from $n=$ integer value to $n=$ non integer value.

\section{PRELIMINEARIES}

Definition2.1: Ishikawa Iteration [8]: Let $X$ be a subset of real or complex numbers and $f: X \rightarrow X$ for $x_{0} \in X$, we have the sequences $\left\{x_{n}\right\}$ and $\left\{y_{n}\right\}$ in $\mathrm{X}$ in the following manner:

$$
\begin{aligned}
& y_{n}=s_{n}^{\prime} f\left(x_{n}\right)+\left(1-s_{n}^{\prime}\right) x_{n} \\
& x_{n+1}=s_{n} f\left(y_{n}\right)+\left(1-s_{n}\right) x_{n}
\end{aligned}
$$

where $0 \leq s_{n}^{\prime} \leq 1, \quad 0 \leq s_{n} \leq 1$ and $\quad s_{n}^{\prime} \quad \& \quad s_{n}$ are both convergent to non zero number.

Definition 2.2 [14]: The sequences $x_{n}$ and $y_{n}$ constructed above is called Ishikawa sequences of iterations or Relative Superior sequences of iterates. We denote it by $R S O\left(x_{0}, s_{n}, s_{n}^{\prime}, t\right)$.

Notice that $R S O\left(x_{0}, s_{n}, s_{n}^{\prime}, t\right)$ with $s_{n}^{\prime}=1$ is $S O\left(x_{0}, s_{n}, t\right)$ i.e. Mann's orbit and if we place $s_{n}=s_{n}^{\prime}=1 \quad$ then $\quad R S O\left(x_{0}, s_{n}, s_{n}^{\prime}, t\right) \quad$ reduces to $O\left(x_{0}, t\right)$.

$$
\text { We remark that Ishikawa orbit }
$$

$R S O\left(x_{0}, s_{n}, s_{n}^{\prime}, t\right)$ with $s_{n}^{\prime}=1 / 2$ is relative superior orbit.

Now we define Mandelbrot sets for function with respect to Ishikawa iterates. We call them as Relative Superior Mandelbrot sets

Definition 2.3[14]: Relative Superior Mandelbrot set RSM for the function of the form $Q_{c}(z)=z^{n}+c$, where $n=1$, $2,3,4 \ldots$ is defined as the collection of $c \in C$ for which the orbit of 0 is bounded i.e. $R S M=\left\{c \in C: Q_{c}^{k}(0): k=0,1,2 \ldots\right\}$ is bounded. 
In functional dynamics, we have existence of two different types of points. Points that leave the interval after a finite number are in stable set of infinity. Points that never leave the interval after any number of iterations have bounded orbits. So, an orbit is bounded if there exists a positive real number.

The collection of points that are bounded, i.e. there exists $\mathrm{M}$, such that $\left|Q^{n}(z)\right| \leq M$, for all $n$, is called as a prisoner set while the collection of points that are in the stable set of infinity is called the escape set. Hence, the boundary of the prisoner set is simultaneously the boundary of escape set and that is Julia set for Q.

Definition 2.4[3]: The set of points RSK whose orbits are bounded under relative superior iteration of the function $Q$ (z) is called Relative Superior Julia sets. Relative Superior Julia set of Q is boundary of Julia set RSK.

2.1 Generation Process[5, 6]: The basic principle of generating fractals employs the iterative formula: $z_{n+1} \leftarrow f\left(z_{n}\right)$ where $z_{0}=$ the initial value of $\mathrm{z}$, and $z_{i}=$ the value of the complex quantity $z$ at the $i$ th iteration. For example, the Mandelbrot's self-squared function for generating fractals is: $f(z)=z^{2}+c$, where $z$ and $c$ are both complex quantities. We propose the use of the transformation function $z \rightarrow z^{n}+c, n \geq 2$ and $z \rightarrow\left(z^{n}+c\right)^{-1}$ for generating fractal images with respect to Ishikawa iterates, where $z$ and $c$ are the complex quantities and $n$ is a real number. Each of these fractal images is constructed as a two-dimensional array of pixels. Each pixel is represented by a pair of $(x, y)$ coordinates. The complex quantities $z$ and $c$ can be represented as:

$$
\begin{aligned}
& z=z_{x}+i z_{y} \\
& c=c_{x}+i c_{y}
\end{aligned}
$$

where $i=\sqrt{(-1)}$ and $z_{x}, c_{x}$ are the real parts and $z_{y} \& c_{y}$ are the imaginary parts of $z$ and $c$, respectively. The pixel coordinates $(x, y)$ may be associated with $\left(c_{x}, c_{y}\right)$ or $\left(z_{x}, z_{y}\right)$.

Based on this concept, the fractal images can be classified as follows: (a) z-plane fractals, wherein $(x, y)$ is a function of $\left(z_{x}, z_{y}\right)$.

(b) c-plane fractals, wherein $(x, y)$ is a

function of $\left(c_{x}, c_{y}\right)$
In the literature, the fractals for $n=2$ in $z$ plane are termed as the Mandelbrot set while the fractals for $n$ $=2$ in $c$ plane are known as Julia sets [10].

\subsection{Generating the fractals:}

$$
\begin{array}{r}
\text { Fractals have been generated from } \\
z \rightarrow z^{n}+c, n \geq 2 \text { and } z \rightarrow\left(z^{n}+c\right)^{-1}, n \geq 2
\end{array}
$$

using escape-time techniques, for example by Gujar etal.[5, 6] and Glynn [7]. We have used in this paper escape time criteria of Relative Superior Ishikawa iterates for both of these functions.

Escape Criterion for Quadratics: Suppose that $|z|>\max \left\{|c|, 2 / s, 2 / s^{\prime}\right\}$, then $\left|z_{n}\right|>(1+\lambda)^{n}|z|$ and $\left|z_{n}\right| \rightarrow \infty$ as $n \rightarrow \infty$.So, $|z| \geq|c|$ and $|z|>2 / s$ as well as $|z|>2 / s^{\prime}$ shows the escape criteria for quadratics.

Escape Criterion for Cubics: Suppose $|z|>\max \left\{|b|,(|a|+2 / s)^{1 / 2},\left(|a|+2 / s^{\prime}\right)^{1 / 2}\right\}$

then $\left|z_{n}\right| \rightarrow \infty$ as $n \rightarrow \infty$. This gives an escape criterion for cubic polynomials

General Escape Criterion: Consider $|z|>\max \left\{|c|,(2 / s)^{1 / n},\left(2 / s^{\prime}\right)^{1 / n}\right\} \quad$ then $\left|z_{n}\right| \rightarrow \infty$ as $n \rightarrow \infty$ is the escape criterion. (Escape Criterion derived in [3] \& [14]).

Note that the initial value $z_{0}$ should be infinity, since infinity is the critical point of $z \rightarrow\left(z^{n}+c\right)^{-1}$. However instead of starting with $z_{0}=$ infinity, it is simpler to start with $z_{1}=c$, which yields the same result. (A critical point of $\mathrm{Z} \rightarrow \mathrm{F}(\mathrm{z})+\mathrm{c}$ is a point where $\mathrm{F}^{\prime}(z)=0$ ). The role of critical points is explained in [1].

\section{GEOMETRY OF RELATIVE SUPERIOR MANDELBROT SETS AND RELATIVE SUPERIOR JULIA SETS:}

The $z$ plane fractal images for the function $z_{n+1} \leftarrow z_{n}^{\alpha}+c$ for positive and negative, both integer and non-integer values of $n$ have been presented by Gujar et al. [5, 6] along with some conjectures about their visual characteristics.

3.1 Z plane fractals: The geometrical analysis of Relative Superior Mandelbrot sets for the function $z \rightarrow z^{n}+c$,

$n \geq 2$ reveals the following changes when we move from the positive integer to a positive non integer value: 


\section{Relative Superior Mandelbrot Sets:}

- Here we notice that the number of lobes in the Relative Superior Mandelbrot sets increases by $(\mathbf{n}+\mathbf{1})$ as one move from an integer to a non integer value nearby the consecutive next integer.

- For odd integer $(n=3)$, we see that there are two self similar lobes initially. As we move to $n=3.2$, there exists a small growth between two major lobes, on the left hand side, thus creating asymmetry along the $\mathrm{Y}$ axis, however the symmetry is retained for the $\mathrm{X}$ axis. When the value of $\mathrm{n}$ increases to 3.6, we predicted the emergence of a small embryonic lobe. This develops more when we move to $\mathrm{n}=3.8$. Thereafter, it develops to a complete self similar lobe at $n=4$. The generalization $(n=15)$ of this result also presents the same picture of development of embryonic lobe (See Figures in Section 4.1). Thus, the Relative Superior Mandelbrot sets are symmetrical only about the real axis.

- For even integer $(n=4)$, we observe that the size of the major lobe situated on the left hand side (negative axis) is increased. In addition, the major lobe appears to be composed of the two partially overlapping lobes in the form of a composite lobe (At $n=4.2$ ). This composite lobe has two constituent lobes that are visible to us on the verge of splitting two major lobes (At $n=4.6$ ). Thus at $\mathrm{n}=4.8$, two self similar lobes appears (See Figures in Section 4.2). The generalization of this result is obtained for $\mathbf{n = 1 6}$, where the growth of the embryonic lobe takes place in the same manner. Here also, the Relative Superior Mandelbrot sets are found symmetrical only about the real axis.

- We studied the geometrical analysis of Relative Superior Mandelbrot for the function $z \rightarrow z^{n}+c, n \geq 2$ as the stable points of this function are black colored. Here the stable region is bounded by the unstable region.

3.2 C plane fractals: The geometrical analysis of Relative Superior Julia sets for the function $z \rightarrow\left(z^{n}+c\right)^{-1}$, $n \geq 2$ reveals the following changes when we move from the positive integer to a positive non integer value:

\section{Relative Superior Julia Sets:}

- Geometrical analysis of the Relative Superior Julia sets of inverse function for non integer values shows that the boundary of the fixed point region forms a $(\boldsymbol{n}+2)$ hypocycloid instead of $(\mathbf{n + 1})$. A hypocycloid is a curve formed by rolling a smaller circle inside a larger circle and tracing a fixed point on the circumference of the smaller circle. The radius of the outer fixed circle for hypocycloid can be computed as $|z|+\left|z^{-n}\right|$, where z satisfies the condition $|z|>n^{1 /(n+2)}$, resulting in a radius of $(n+2) n^{-n /(n+2)}$. The radius of inner moving circle is $\left|z^{-n}\right|$ yielding $n^{-n /(n+2)}$.

- The inverse function for the odd integer $(n=3)$, shows that the space between the legs emerges at $n=3.2$. Further, a thin leg appears at $n=3.6$, which gets broaden as self similar legs at $n=3.8$. So, with the change from the integer to the non integer value the symmetry about both $\mathrm{X}$ and $\mathrm{Y}$ axes, now changes to only about $\mathrm{X}$ axis. Further only the rotational symmetry is maintained while the reflection symmetry is loosed at non integer values (See Figures in Section 5.1).

- The Relative Superior Julia sets for the even integer $(n=$ 4), we observe that the size of the major lobe situated on the left hand side (negative axis) is increased. In addition, the major lobe appears to be composed of the two partially overlapping lobes in the form of a composite lobe (At $n=4.2$ ). This composite lobe has two constituent lobes that are visible to us on the verge of splitting two major lobes (At $n=4.6$ ). Further at $n=4.8$, an embryonic self similar lobe develops. Thus the symmetry is maintained only along the $X$ axis for non integer values (See Figures in Section 5.2).

- The generalization of the results is obtained for odd and even values of $n$ can be observed for $n=15$ and $n=16$, where the growth of the embryonic lobe takes place in the same manner. Here the structure resembles to that of a constellation similar to planetary arrangement with central circular planet surrounded by satellite like structures. Furthermore, the size of the central planet reduces as the value of $\boldsymbol{n}$ reduces, while the satellite structures enlarge and diffuses. On the other hand, with the increase in the value of $\boldsymbol{n}$, the central planet size increases and more satellite structures appears with very small radius (See Figures in Section 5.3).

- The planetary arrangement with central circular planet surrounded by satellite like structures describes reflection as well as rotational symmetry.

- It is also observed for the planetary arrangement with central circular planet that, the stable region is represented by light color which surrounds the constellation while the dark color regions represent the unstable areas. This situation is reverse of the situation

for the fractals of the function $z \rightarrow z^{n}+c, n \geq 2$ where the stable regions are bounded by unstable regions. 


\section{GENERATION OF RELATIVE SUPERIOR MANDELBROT SETS}

We present here some Relative Superior Mandelbrot sets for the function $z \rightarrow z^{n}+c, \quad n \geq 2$, for integer and some non integer values.

4.1 Relative Superior Mandelbrot Sets for Cubic function

Figure 1: Relative Superior Mandelbrot Set for $s=0.8$, $\mathrm{s}^{\prime}=\mathbf{0 . 3}, \mathrm{n}=\mathbf{3}$

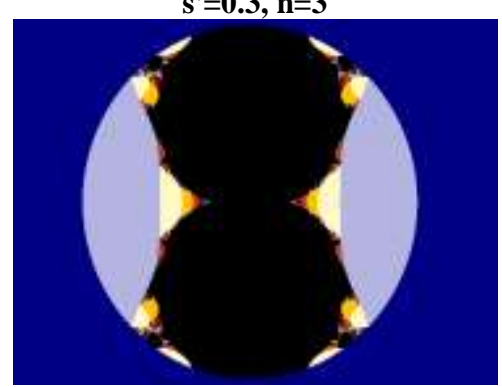

Figure 2: Relative Superior Mandelbrot Set for $s=0.8$, $\mathrm{s}^{\prime}=0.3, \mathrm{n}=3.2$

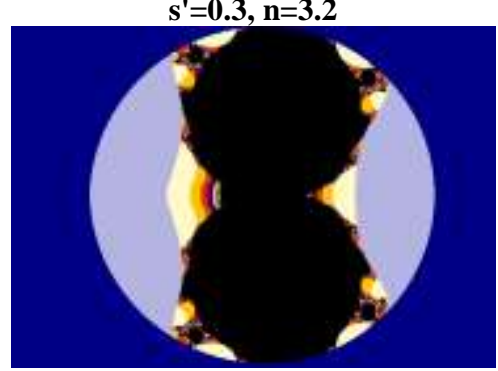

Figure 3: Relative Superior Mandelbrot Set for $\mathrm{s}=\mathbf{0 . 8}$,

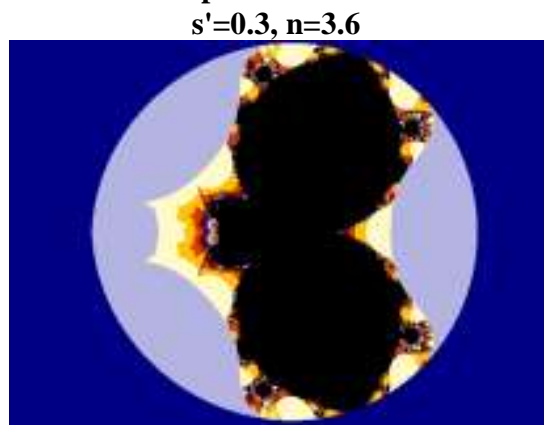

Figure 4: Relative Superior Mandelbrot Set for $s=0.8$, $\mathrm{s}^{\prime}=0.3, \mathrm{n}=3.8$

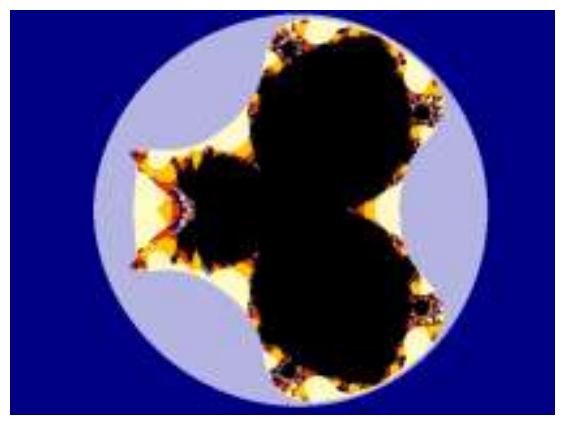

4.2 Relative Superior Mandelbrot Sets for Bi-quadratic function:

Figure 1: Relative Superior Mandelbrot Set for $s=0.5$, $\mathrm{s}^{\prime}=\mathbf{0 . 4}, \mathrm{n}=4$

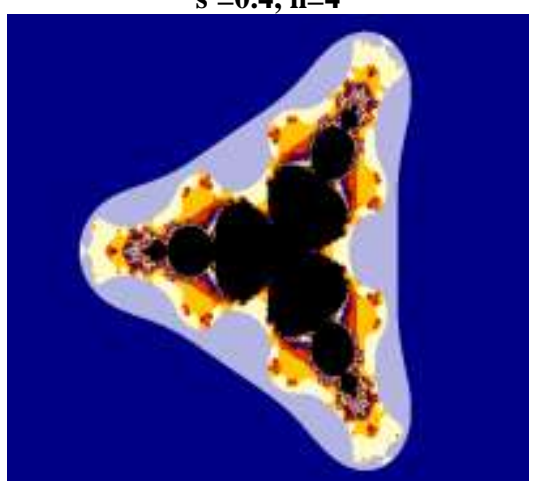

Figure 2: Relative Superior Mandelbrot Set for $\mathrm{s}=\mathbf{0 . 5}$, $\mathrm{s}^{\prime}=\mathbf{0 . 4}, \mathrm{n}=4.2$

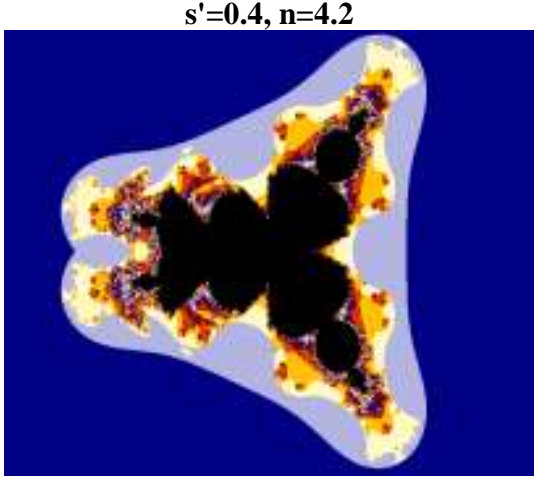

Figure 3: Relative Superior Mandelbrot Set for $\mathrm{s}=\mathbf{0 . 5}$, $s^{\prime}=0.4, n=4.6$

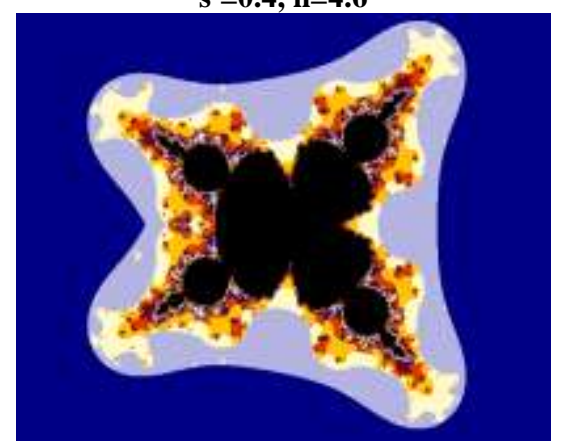

Figure 4: Relative Superior Mandelbrot Set for $\mathrm{s}=\mathbf{0 . 5}$, $s^{\prime}=0.4, n=4.8$

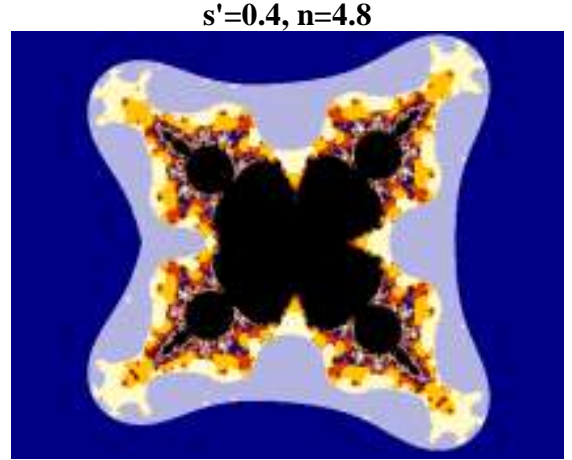

4.3 Generalization of Relative Superior Mandelbrot Set: 
Figure 1: Relative Superior Mandelbrot Set for $s=0.4$,

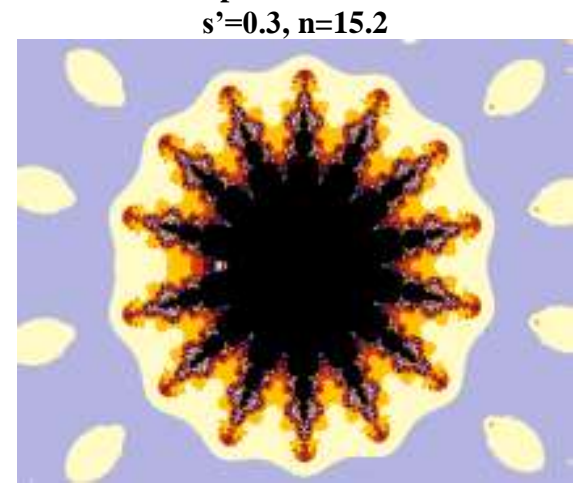

Figure 2: Relative Superior Mandelbrot Set for $\mathrm{s}=\mathbf{0 . 4}$,

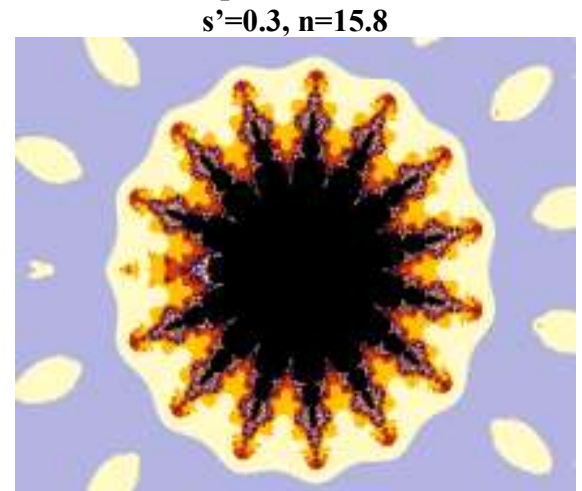

Figure 3: Relative Superior Mandelbrot Set for $\mathrm{s}=\mathbf{0 . 5}$,

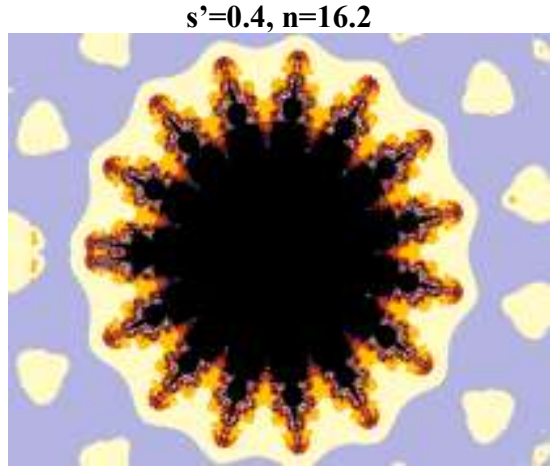

Figure 4: Relative Superior Mandelbrot Set for $\mathrm{s}=\mathbf{0 . 5}$,

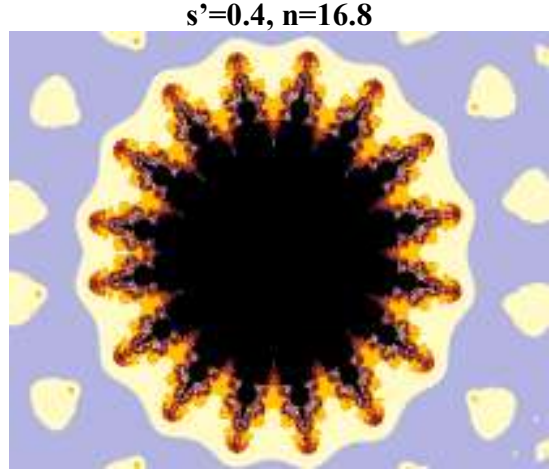

\section{GENERATION OF RELATIVE SUPERIOR JULIA SETS:}

We present here some Relative Superior Julia Sets for the function $z \rightarrow\left(z^{n}+c\right)^{-1}, n \geq 2$ for integer and some non integer values. Here stable points are light colored while unstable are dark colored regions.

\subsection{Relative Superior Julia sets for Cubic function:}

Figure 1: Relative Superior Julia Set for $s=0.5, s^{\prime}=0.4$,

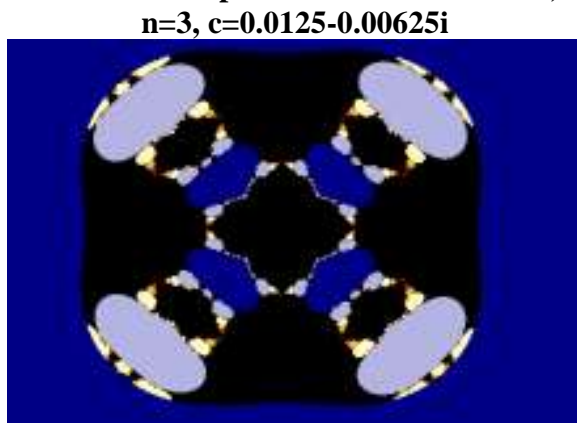

Figure 2: Relative Superior Julia Set for $s=0.5, \mathrm{~s}^{\prime}=0.4$, $\mathrm{n}=3.2, \mathrm{c}=0.03488180321-0.01680604855 \mathrm{i}$

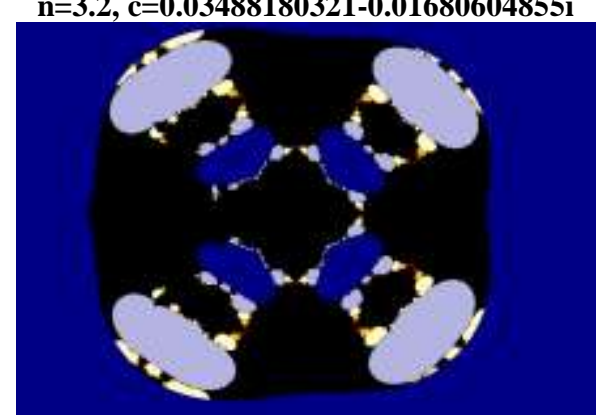

Figure 3: Relative Superior Julia Set for $s=0.5, s^{\prime}=0.4$,

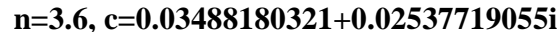

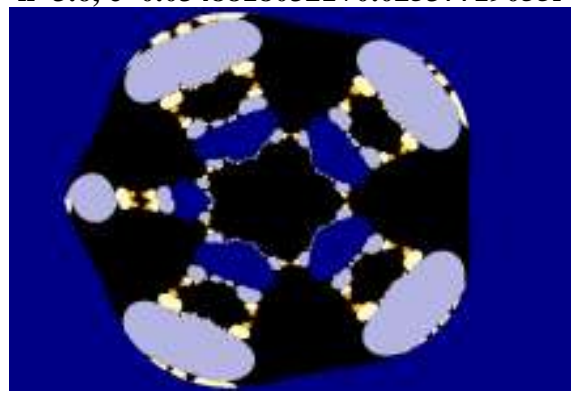

Figure 4: Relative Superior Julia Set for $s=0.5, s^{\prime}=0.4$,

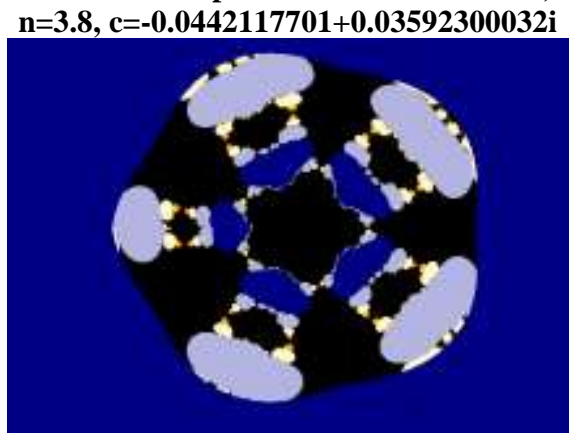

5.2 Relative Superior Julia sets for Bi-quadratic function:

Figure 1: Relative Superior Julia Set for $s=0.8, s^{\prime}=0.2$, $n=4, c=0.0125+0 i$ 


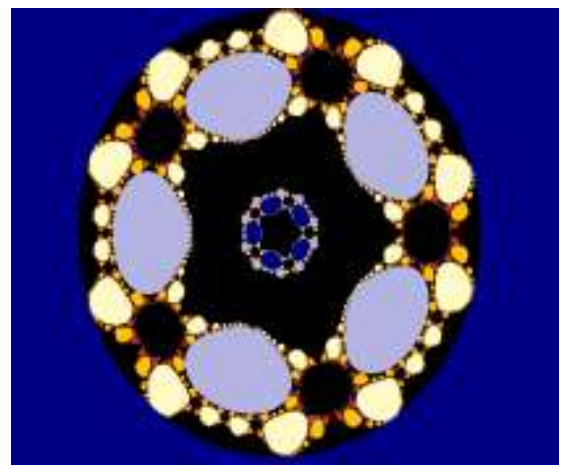

Figure 2: Relative Superior Julia Set $s=0.8, s^{\prime}=0.2$, $\mathrm{n}=4.2, \mathrm{c}=\mathbf{0 . 0 4 0 1 5 4 7 0 7 9 8 + 0 . 0 3 5 9 2 2 9 9 9 6 3 \mathrm { i }}$

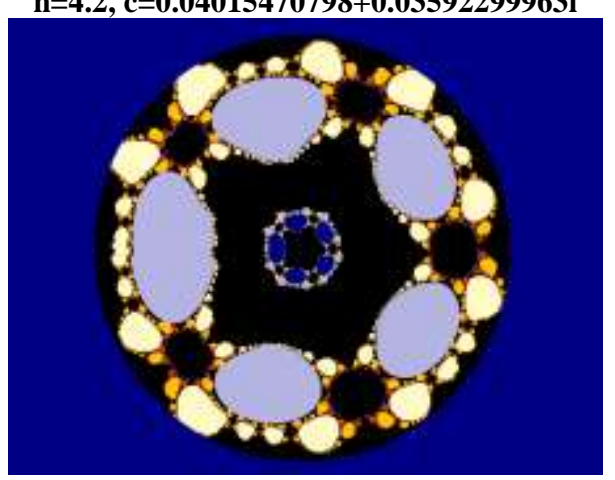

Figure 3: Relative Superior Julia Set for $s=0.8, s^{\prime}=0.2$, $\mathrm{n}=4.6, \mathrm{c}=0.003244373774+0.02010428497 \mathrm{i}$

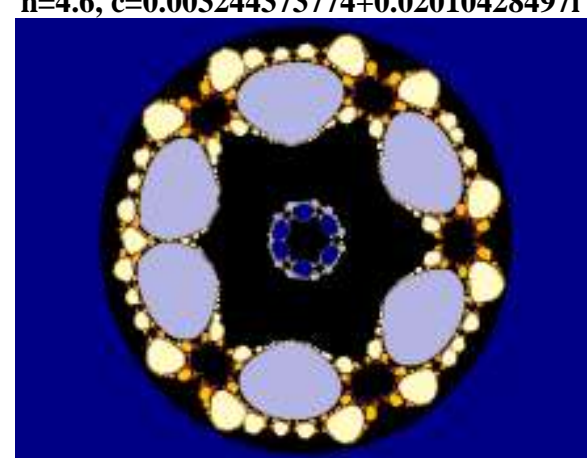

Figure 4: Relative Superior Julia Set $s=0.8, s^{\prime}=0.2$, $\mathrm{n}=4.8, \mathrm{c}=-\mathbf{0 . 0 1 7 8 4 7 2 4 5 7 7 + 0 . 0 3 0 6 5 0 0 9 4 7 4 i}$

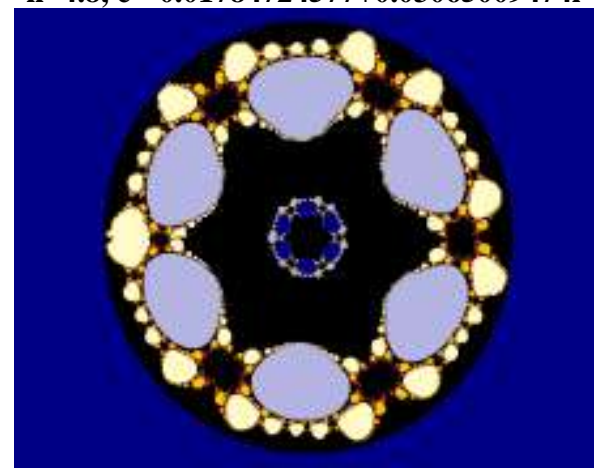

5.3 Generalization of Relative Superior Julia Set:

Figure 1: Relative Superior Julia Set for $s=0.5, s^{\prime}=0.4$,

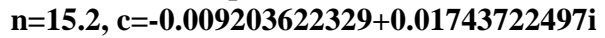

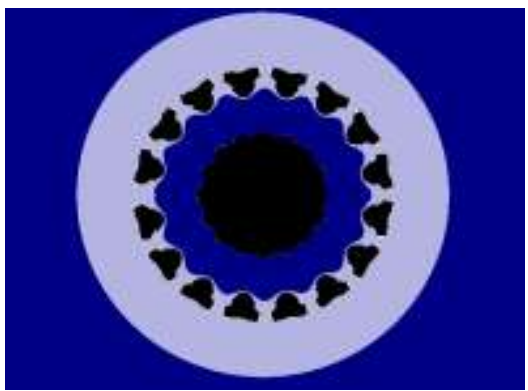

Figure 2: Relative Superior Julia Set for $\mathrm{s}=\mathbf{0 . 5}, \mathrm{s}^{\prime}=\mathbf{0 . 4}$,

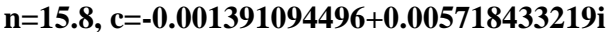

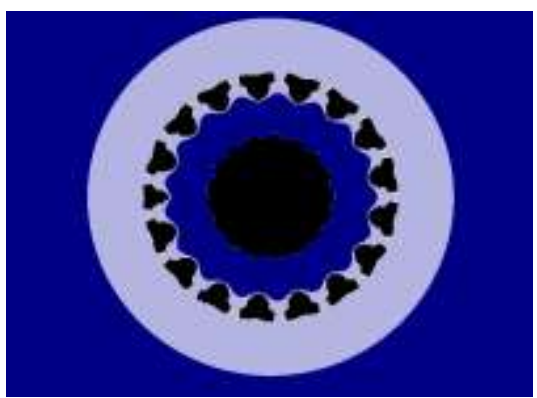

Figure 3: Relative Superior Julia Set for $s=0.5, s^{\prime}=0.4$,

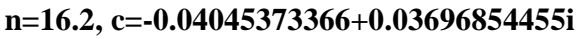

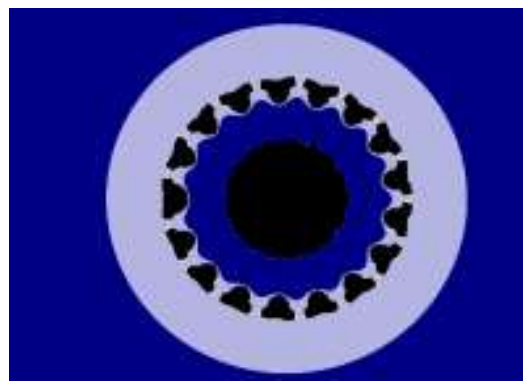

Figure 4: Relative Superior Julia Set for $s=0.5, s^{\prime}=0.4$, $\mathrm{n}=16.8, \mathrm{c}=-\mathbf{0 . 0 6 3 8 9 1 3 1 7 1 5 + 0 . 0 2 1 3 4 3 4 8 8 8 8 \mathrm { i }}$

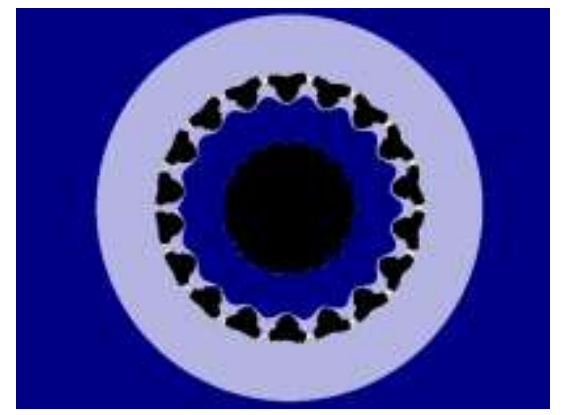

\section{GRAPHS}

7.1 Graphs for the function $z \rightarrow z^{n}+c, n \geq 2$ : 
Figure 1: Orbit of $\mathrm{F}(\mathrm{z}) \mathrm{s}=\mathbf{0 . 8}, \mathrm{s}^{\prime}=\mathbf{0 . 2}, \mathbf{n}=\mathbf{3 . 6}$, $c=0.006067272682+0.02897841648 i$

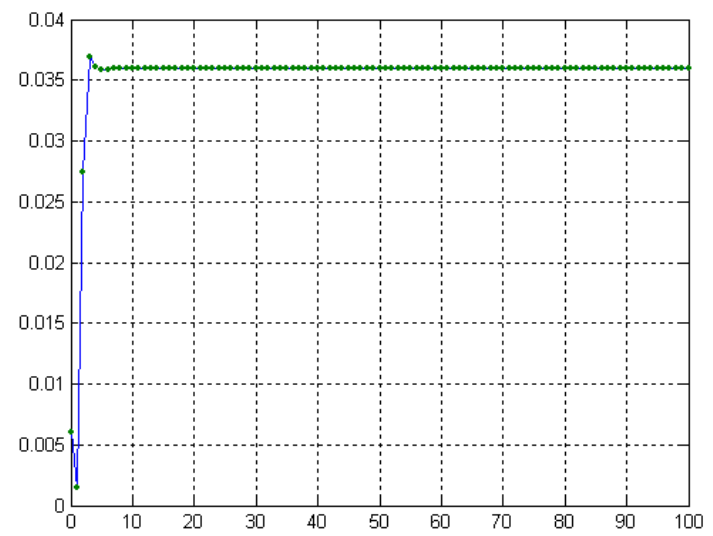

Figure 2: Orbit of $F(z) s=0.5, s^{\prime}=0.4, n=4.8$, $c=-0.1125374895-0.0520056533 i$

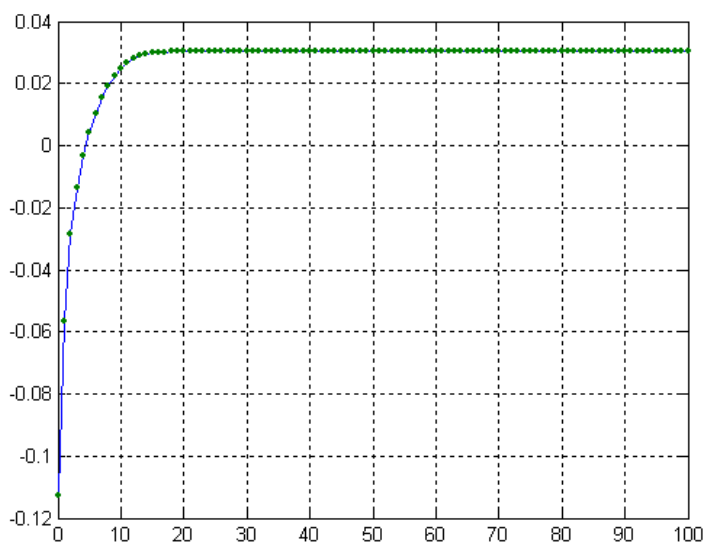

7.2 Graphs for the function $z \rightarrow\left(z^{n}+c\right)^{-1}, n \geq 2$ :

Figure 1: Orbit of $\mathrm{F}\left(\mathrm{z} s=\mathbf{0 . 5}, \mathrm{s}^{\prime}=\mathbf{0 . 4}, \mathrm{n}=\mathbf{3 . 2}\right.$, $c=0.03488180321-0.01680604855 i$

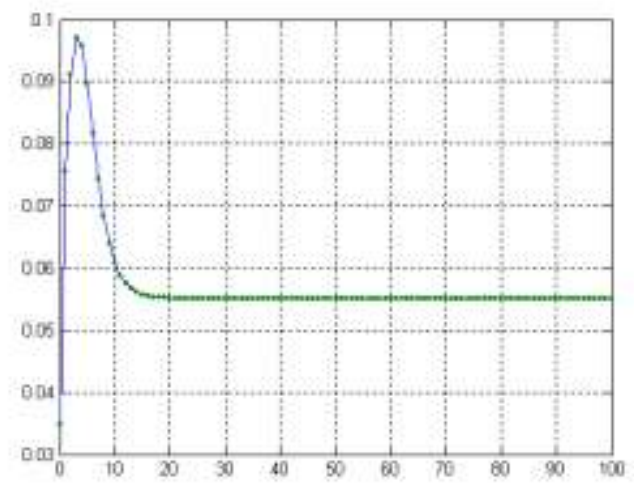

Figure 2: Orbit of $F(z) s=0.8, s^{\prime}=0.2, n=4.6$, $c=0.003244373774+0.02010428497 i$

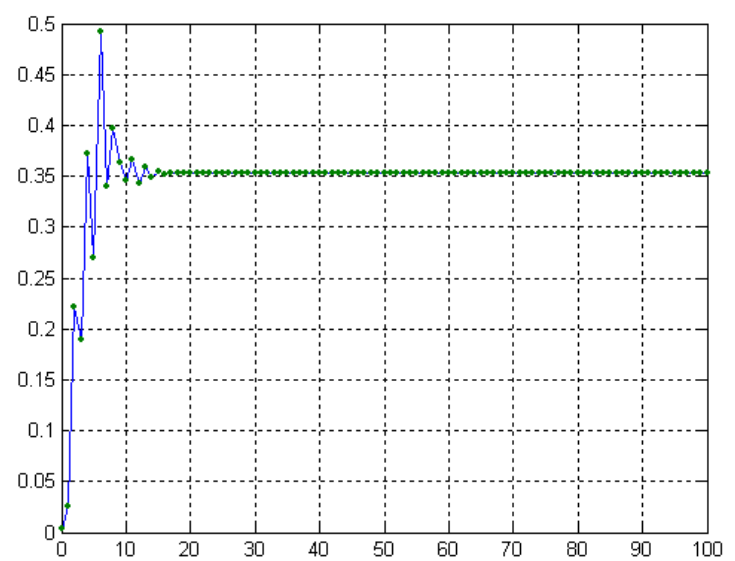

\section{CONCLUSION}

In this paper, we have considered the transformation of the functions $z \rightarrow z^{n}+c, n \geq 2$ and $z \rightarrow\left(z^{n}+c\right)^{-1}$, $n \geq 2$. We mathematically analyzed the visual characteristics of the fractal images in the complex $z$ and $c$ planes respectively. The $\mathrm{Z}$ plane fractal images for the function $z \rightarrow z^{n}+c, n \geq 2$ showed that the stable region is bounded by unstable region. Besides this, the non integer value change brought the embryonic structure in the form of lobe. On the other hand the $\mathrm{C}$ plane geometrical analysis of the function $z \rightarrow\left(z^{n}+c\right)^{-1}, \quad n \geq 2$ represented the planetary type structure comprising of central planet with satellites. Here non integer value change showed the embryonic self similar growth in the satellite pattern. Also, this function exhibits the unstable region embedded within the stable region.

\section{REFERENCES}

[1] B. Branner, "The Mandelbrot Set", Proceedings of Symposia in Applied Mathematics39 (1989), 75-105.

[2] P. Blanchard, "Complex Analytic Dynamics on the Riemann Sphere", Bulletin of the American Mathematical Society 11, 1 (1984), 85-141.

[3] Y. S. Chauhan, R. Rana and A. Negi, New Tricorns and Multicorns of Ishikawa Iterates, In Press, Int. Journal of Computer Application (Oct. 2010 Edition

[4] S. Dhurandar, V. C. Bhavsar and U. G. Gujar, "Analysis of z-plane fractal images from $z \leftarrow z^{\alpha}+c$ for $\alpha<0$ ", Computers and Graphics 17, 1 (1993), 89-94.

[5] U. G. Gujar and V. C. Bhavsar, "Fractals from $z \leftarrow z^{\alpha}+c$ in the Complex c-Plane", Computers and Graphics 15, 3 (1991), 441-449. 
[6] U. G. Gujar, V. C. Bhavsar and N. Vangala, "Fractals from $z \leftarrow z^{\alpha}+c$ in the Complex z-Plane", Computers and Graphics 16, 1 (1992), 45-49.

[7] E. F. Glynn, "The Evolution of the Gingerbread Mann", Computers and Graphics 15,4 (1991), 579582.

[8] S. Ishikawa, "Fixed points by a new iteration method", Proc. Amer. Math. Soc.44 (1974), 147-150.

[9] G. Julia, "Sur 1' iteration des functions rationnelles", J Math Pure Appli. 8 (1918), 737-747

[10] K. W. Shirriff, “An investigation of fractals generated by $z \rightarrow z^{-n}+c$, , Computers and Graphics 13, 4 (1993), 603-607.
[11] B. B. Mandelbrot, "The Fractal Geometry of Nature", W. H. Freeman, New York, 1983.

[12] H. Peitgen and P. H. Richter, "The Beauty of Fractals", Springer-Verlag, Berlin,1986.

[13] C. Pickover, "Computers, Pattern, Chaos, and Beauty”, St. Martin's Press, NewYork, 1990.

[14] R. Rana, Y. S. Chauhan and A. Negi, Non Linear dynamics of Ishikawa Iteration, In Press, Int. Journal of Computer Application (Oct. 2010 Edition).

[15] S. T. Welstead and T. L. Cromer, "Coloring Periodicities of Two-dimensional Mappings", Computers and Graphics 13, 4 (1989), 539-543. 